\title{
Standardization and Updating of a Drug Allergy Testing Program: The McGill Experience and Impact on Pharmacy Activities
}

\author{
Gilbert Matte, Joseph Shuster, Chantal Guevremont, Phil Gold, Fabrice Leong, Zinquon Ngan, \\ André Bonnici, and Chris Tsoukas
}

\section{INTRODUCTION}

Datients' claims of drug allergies are frequent but often not 1 confirmed. ${ }^{1}$ An unsubstantiated drug allergy label on a patient's chart can have a significant impact on clinical management. Retrospective studies have revealed that 3\% to $6 \%$ of admissions to health care institutions are for unpredictable drug reactions, including drug allergies. ${ }^{2}$ The consequences of unresolved drug allergy claims for patient care are substantial, including treatment delays, use of suboptimal treatment, longer hospital stays, and greater risks of complications. ${ }^{3-7}$

Drug allergy testing is effective for "de-labelling" individuals with suspected drug allergies; however, testing methods have often varied between, and even within, health care institutions. Until 2017, there was variability in testing protocols used in the McGill University Health Centre (in Montréal, Quebec). Variations between physicians were high, and the protocols were derived using available literature and were not always updated. ${ }^{8,9}$ Allergists used sequential challenge doses set between 2- and 10-fold increments given at variable times (typically $30-45 \mathrm{~min}$ between challenge doses). ${ }^{8-13}$ The lack of standardization made it difficult for the pharmacy to provide efficient compounding and clinical support. Literature comparing the various protocols was not available, which made it difficult for the pharmacists to either accept or reject the doses of prescriptions as written. It was clear that local policies and drug allergy testing algorithms had to be either created or updated. A comprehensive, focused drug evaluation program was inaugurated. Critical to implementation of the initiative was the creation of an allergist-pharmacist team to develop standard operating procedures, consensus-based uniform testing protocols, documentation forms, predefined prescriptions, and reports.
A critical component of any drug allergy evaluation is risk management. To that end, a drug allergy risk assessment tool was developed and its application made mandatory before any allergy testing. This tool had the dual aims of not only avoiding the testing of patients with absolute contraindications to drug challenge, but also identifying patients who could undergo single-dose, low-risk challenge.

This manuscript outlines the steps taken to establish the new drug allergy testing program and describes the subsequent positive impact in terms of streamlining pharmacy activity and improving patients' access to drug allergy testing.

\section{DESCRIPTION OF THE DRUG ALLERGY PROGRAM}

The Division of Allergy and Clinical Immunology at the McGill University Health Centre provides quaternary care and conducts research on and teaching about drug allergy. On average, 6000 patients with potential allergy (to drugs as well as food) are evaluated annually. The drug allergy screening program was gradually modified and fully implemented in 2017. As such, allergy testing transitioned from a physician-specific clinic to a program offered every day of the week, independent of the physician. Originally, prescriptions for testing of the same suspected drug allergy varied among physicians, and extensive compounding was required. To demonstrate these differences, 93 prescriptions for allergy challenge were reviewed; from these, the 5 oral medications with the most prescribing variability were identified. The prescriptions for these 5 medications, written by 8 physicians, revealed no consistent prescribing patterns (Table 1). The approach for drug allergy testing was to perform skin tests and then perform a graded drug challenge if appropriate. 
This single copy is for your personal, non-commercial use only.

For permission to reprint multiple copies or to order presentation-ready copies for distribution, contact CJHP at publications@cshp.ca

Table 1. Prescribing Variations within and between Physicians for 5 Most Commonly Prescribed Drugs Tested for Allergies before 2011

Drug Tested; Variant Prescription Sequences for Allergy Challenge

\begin{tabular}{|c|c|c|c|c|c|}
\hline \multirow[b]{2}{*}{ Physician } & \\
\hline & ASA & Celecoxib & Ibuprofen & Moxifloxacin & Penicillin V \\
\hline 1 & NA & $\begin{array}{c}1,10,30,60 \mathrm{mg} \\
2,10,50,100 \mathrm{mg} \\
\text { plus } 5 \times \text { placebo } \\
10,30,60 \mathrm{mg} \\
2,5,10,25 \mathrm{mg} \text { plus } \\
4 \times \text { placebo } \\
1,50,50,100 \mathrm{mg} \\
\text { plus } 2 \times \text { placebo }\end{array}$ & $2,4,20,100 \mathrm{mg}$ & NA & NA \\
\hline 2 & $\begin{array}{l}15,25,100,325 \mathrm{mg} \\
\text { plus } 2 \times \text { placebo } \\
1,81 \mathrm{mg} \text { plus } 1 \times \text { placebo } \\
25,50,100,200,325 \mathrm{mg} \\
5,50,100,325 \mathrm{mg}\end{array}$ & $\begin{array}{l}2,20,200 \mathrm{mg} \\
20,200 \mathrm{mg} \\
20,200 \mathrm{mg} \\
1,20,200 \mathrm{mg}\end{array}$ & $\begin{array}{c}0.2,2,20,200 \mathrm{mg} \\
0.2,5,50,200 \mathrm{mg} \\
\text { plus } 2 \times \text { placebo }\end{array}$ & $\begin{array}{c}0.4,4,40,400 \mathrm{mg} \\
\text { plus } 1 \times \text { placebo }\end{array}$ & $\begin{array}{c}3,30,300 \mathrm{mg} \\
\text { plus } 2 \times \text { placebo } \\
3,30,300,300 \mathrm{mg} \\
100,200,300 \mathrm{mg} \\
\text { plus } 1 \times \text { placebo }\end{array}$ \\
\hline 3 & $\begin{array}{l}32.5,325 \mathrm{mg} \\
20,40,325 \mathrm{mg} \\
1,5,10,20,80,160 \\
325 \mathrm{mg} \text { plus } 1 \times \\
\text { placebo }\end{array}$ & $20,200 \mathrm{mg}$ & $40,400 \mathrm{mg}$ & $40,400 \mathrm{mg}$ & $30,300 \mathrm{mg}$ \\
\hline 4 & $\begin{array}{l}5,325 \mathrm{mg} \text { plus } 1 \times \\
\text { placebo } \\
1,5,80,160,325 \mathrm{mg}\end{array}$ & $\overline{N A}$ & $5,10,25 \mathrm{mg}$ & $\begin{array}{l}400 \mathrm{mg} \text { plus } \\
4 \times \text { placebo } \\
4,40,200 \mathrm{mg} \text { plus } \\
8 \times \text { placebo }\end{array}$ & NA \\
\hline 5 & $\begin{array}{l}3.25,32.5,325 \mathrm{mg} \\
1,10,100 \mathrm{mg}\end{array}$ & NA & NA & $400 \mathrm{mg}$ & $\begin{array}{l}30,300 \mathrm{mg} \\
300 \mathrm{mg}\end{array}$ \\
\hline$\overline{6}$ & $\mathrm{NA}$ & $\begin{array}{c}2,4,10,80,100 \mathrm{mg} \\
\text { plus } 5 \times \text { placebo } \\
20,200 \mathrm{mg} \text { plus } \\
2 \times \text { placebo }\end{array}$ & NA & NA & NA \\
\hline 7 & NA & NA & NA & NA & $\begin{array}{l}100,200,300 \mathrm{mg} \\
100,200,300 \mathrm{mg} \\
300 \mathrm{mg}\end{array}$ \\
\hline 8 & $325 \mathrm{mg}$ & NA & NA & NA & $300 \mathrm{mg}$ \\
\hline
\end{tabular}

Most allergists independently assessed and tested their own patients without sharing their protocols. To meet the increased demand for testing and to optimize pharmacist support without additional resources, the interdisciplinary team created a novel tool, established clear policies, attained protocol consensus, and generated standardized procedures. Following this standardization, drug challenges were conducted daily, and supervision of the challenges was pooled among allergists to allow flexibility in booking. The institutional research ethics board determined that the study was acceptable, but formal ethics approval was not required.

\section{POLICIES AND PROCEDURES}

On the basis of published guidelines, the emerging literature. ${ }^{14,15}$ and the allergists' clinical experiences, an internal algorithm was drafted (Figure 1). Policies and standard operating procedures were then developed. The policies defined the roles of clinic physicians, pharmacists, and allergy nurses in conducting challenge and desensitization of suspected drug allergies. They also established specific safe venues for the testing to be done, human resources, and conditions for safe performance of the tests. Table 2 lists and briefly describes the documents generated.
Existing reports and consent forms were reviewed and improved to be more informative.

A local risk assessment tool was also developed for use in patient triage, whereby patients with suspected drug allergy were stratified according to risk. The triage tool ensured uniformity in risk assessment by all allergists, eliminating patients at high risk for anaphylactic or other serious reactions. The nature of the challenge (single dose or multiple doses) was thus better defined, which enabled the pharmacy to have a consistent approach to compounding protocols while keeping the protocols specific for each drug. With this standardization, physicians could crosssupervise drug challenges ordered by their colleagues. The shared evaluation document led to rapid sharing of historical information by both the nursing staff and the allergist supervising the procedure. Since this study was conducted, an electronic version of the risk assessment tool has been developed (examples of such tools for penicillin are available in the literature and can also be used for other drugs $\left.{ }^{16}\right)$. Additional information about both the original tool and the newer electronic version are available, upon request to the corresponding author, to other institutions that are interested in developing their own tools. 


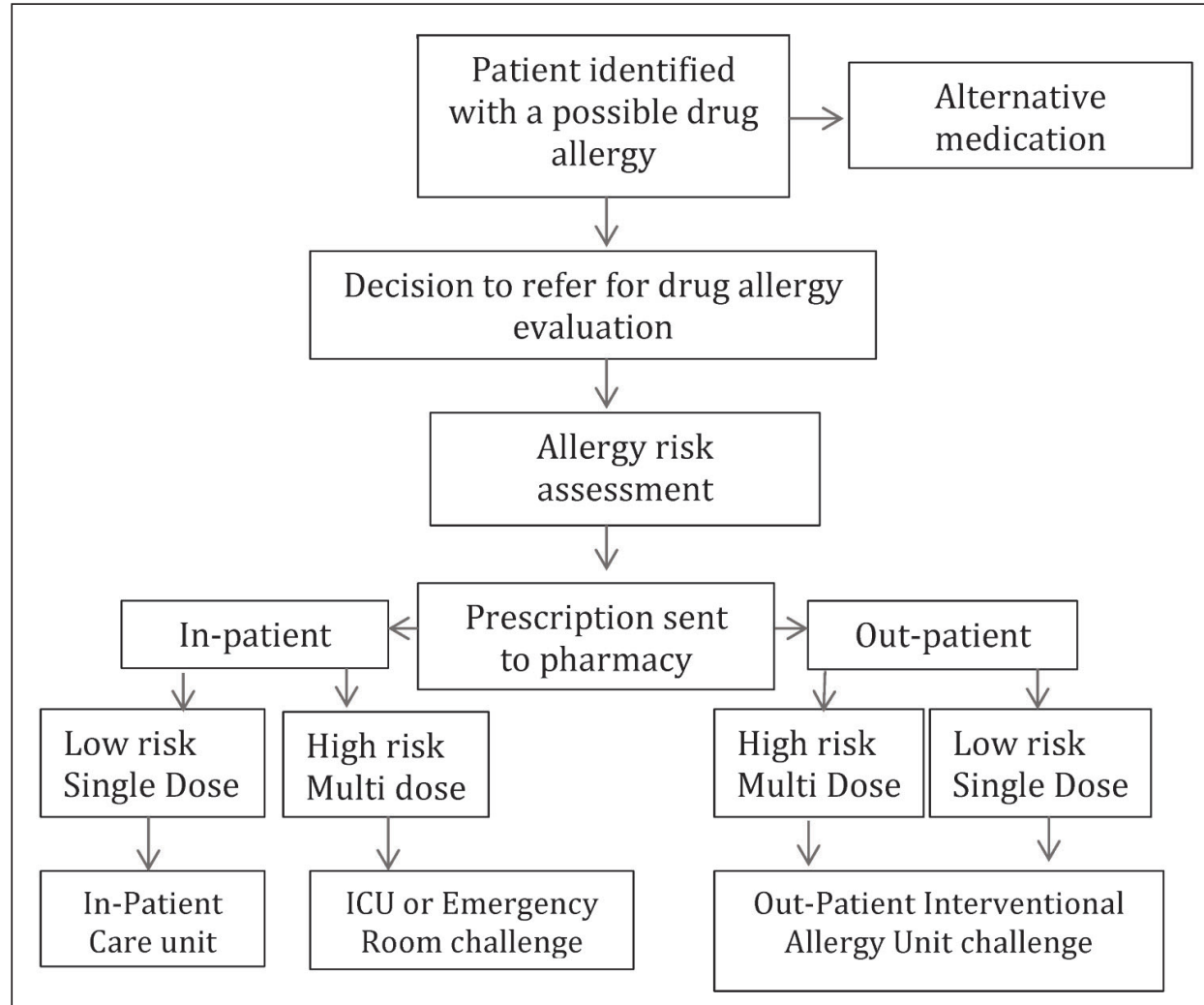

Figure 1. Allergy provocation testing algorithm. ICU = intensive care unit.

For drug provocation, doses for the "higher-risk" patients were limited to $1 \%, 10 \%, 30 \%$, and $100 \%$ of the recommended single drug dose. Observation times for oral drugs before administration of the next dose remained at 30-45 min. All patients were monitored for at least $1 \mathrm{~h}$ after receiving the last dose of the sequence. For lower-risk patients, a single dose of a commercially available formulation was administered, either as an entire tablet or as a capsule. If a "blinded" dose was requested, a crushed drug preparation was placed in an empty capsule. Compounding was performed according to provincial requirements, using only drugs licensed by Health Canada.

A prescription was required for each challenge. Prescriptions were sent to the pharmacy and individually documented. The drugs identified as those most commonly tested as a single dose were provided as floor stock and registered retrospectively in the database. Other, less common drug requests for single and compounded preparations were sent to the clinic upon the patient's arrival. All suspected drug allergies were entered in the allergy module of the electronic medical record (EMR) and flagged until testing occurred with the comment "suspected allergy, as per allergy testing request, results pending".

The tests were performed either in a dedicated interventional outpatient allergy facility or on an inpatient care unit. If no adverse reaction was observed after the last dose, the patient was discharged home and asked to call the next day to report any late reactions. Current literature and guidelines suggested that multiple sequential doses on the same day could lead to desensitization rather than providing diagnostic information. ${ }^{8,14}$ Therefore, the daily number of testing doses was limited to a maximum of 2 (not including placebo). The evaluation and all testing were carried out under the direct supervision of trained allergists.

\section{EVALUATION OF THE PROGRAM}

Pharmacy compounding records were used to review prescribing patterns before and after program initiation. Pharmacy resource utilization was assessed using variables captured in the database, including the number of prescriptions, the drugs and doses requested, and pharmacy activities related to the drug testing program. To estimate and normalize the compounding activities, the monthly number of doses dispensed was divided by the number of prescriptions, and the ratio of doses per prescription was obtained. The results were compared for the same 12-month calendar period before standardization (July 2014 to June 2015) and after full standardization (July 2016 to June 2017). The 12-month transition period (July 2015 to June 2016) was excluded to eliminate any carry-over effect.

\section{RESULTS}

Patients were tested for a total of 128 drugs, most belonging to the antibiotic and nonsteroidal anti-inflammatory drug 
This single copy is for your personal, non-commercial use only.

For permission to reprint multiple copies or to order presentation-ready copies for distribution, contact CJHP at publications@cshp.ca

Table 2. Documents Related to Drug Allergy Testing Reviewed or Newly Created

\begin{tabular}{|c|c|c|}
\hline Document & Description & Status \\
\hline \multicolumn{3}{|l|}{ Policies } \\
\hline Allergy provocation testing & $\begin{array}{l}\text { Guidance in all aspects of allergy provocation } \\
\text { (clarifies who can test and where) }\end{array}$ & New document \\
\hline Drug desensitization & $\begin{array}{l}\text { Guidance in all drug desensitization } \\
\text { (clarifies who can desensitize and where) }\end{array}$ & New policy \\
\hline Mandatory risk assessment before provocation testing & $\begin{array}{l}\text { Policy to ensure that a specific risk assessment is } \\
\text { performed before testing }\end{array}$ & New policy \\
\hline \multicolumn{3}{|l|}{ Standard operating procedures } \\
\hline Main standard operating procedure & General description of the department activities & New document* \\
\hline $\begin{array}{l}\text { Standard operating procedure for allergy } \\
\text { provocation testing }\end{array}$ & General description of provocation testing & New document* \\
\hline Preoperative operating procedure for drug allergy & General description of the pre-op B lactam allergy testing & New document* \\
\hline Desensitization procedures & General description for desensitization & New document* \\
\hline $\begin{array}{l}\text { Standard operating procedure for skin and intradermal } \\
\text { allergy testing }\end{array}$ & General description for skin testing & New document* \\
\hline \multicolumn{3}{|l|}{ Patient information documents } \\
\hline Patient instructions for provocation test & Information to patient & Updated document \\
\hline Consent form for food and /or drug provocation testing & Consent form & Updated document \\
\hline \multicolumn{3}{|l|}{ Standard pharmacy prescriptions } \\
\hline Pharmacy prescription for drug provocation testing & Standard preprinted prescription & New document \\
\hline $\begin{array}{l}\text { Pharmacy prescription for allergy testing } \\
\text { prick test / intra dermal }\end{array}$ & Standard preprinted prescription & New document \\
\hline $\begin{array}{l}\text { Pharmacy prescription for desensitization and } \\
\text { rescue medication }\end{array}$ & Standard preprinted prescription & New document \\
\hline \multicolumn{3}{|l|}{ Nursing orders } \\
\hline Before and after provocation testing & Standard preprinted order & Updated \\
\hline For desensitization & Standard preprinted order & Updated \\
\hline \multicolumn{3}{|l|}{ Reporting forms } \\
\hline Pre drug provocation testing risk assessment & Standard preprinted prescription & New document \\
\hline Provocation test result & Standard preprinted form & Updated \\
\hline Nursing reporting form for food and drug provocation & Standard preprinted form & Updated \\
\hline Physician reporting of drug provocation test & Standard preprinted form & Updated \\
\hline
\end{tabular}

*Previously based on published papers and/or case reports, specific to each physician.

(NSAID) classes. Penicillin V emerged as the most frequently prescribed medication (30\% of prescriptions before and 32\% of prescriptions after standardization), followed by amoxicillin ( $6 \%$ before and 15\% after standardization). Other antibiotics with frequent requests for allergy testing included ciprofloxacin, azithromycin, clindamycin, and sulfamethoxazole-trimethoprim. However, the latter accounted for fewer than 20 prescriptions per year both before and after standardization.

In the second most frequently tested category of drugs, the NSAIDs, ibuprofen, acetylsalicylic acid, celecoxib, and naproxen together accounted for $18 \%$ and $13 \%$ of annual prescriptions before and after standardization, respectively.

Following standardization, several changes were noted. The annual number of prescriptions increased (from 478 for January to December 2015 to 748 for the same time frame in 2017), a 56\% increase. Figure 2 details the monthly volume of prescriptions before and after standardization. The average number of doses per prescription was reduced, and the dose-to-prescription ratio declined from 2.04 to 1.47 (2-tailed $t$ test, $p<0.001$ ). The total annual quantity of doses required remained steady, at about 1050 , while the proportion of single doses, using commercially available formulations, increased from $7 \%$ to $33 \%$. In the first 6 months of 2019, the pharmacy dispensed 595 prescriptions for the purpose of allergy testing, of which 315 (53\%) were dispensed as single-dose challenges.

After standardization, 4 possible dosages were available for predefined prescriptions: $1 \%, 10 \%, 30 \%$, and $100 \%$. The drug allergy testing was performed most commonly as single or 2 divided doses of $10 \%$ and $100 \%$ in a single-day challenge protocol. The $1 \%$ dose was rarely used, and less than $10 \%$ of all prescriptions involved a request for a $30 \%$ dose.

The infrastructure to support drug allergy testing consisted of a single pharmacist and a single technical assistant. Both of these individuals also supported the pharmaceutical research and special access programs. After-hours support for drug allergy testing continues to be limited to inpatients and is handled by the after-hours pharmacy distribution team. Standardization led to development of simpler compounding procedures that permitted easy accessibility after hours. Following standardization there was no increase in personnel to support the drug allergy testing program, despite the monthly increase in volume of prescriptions. 


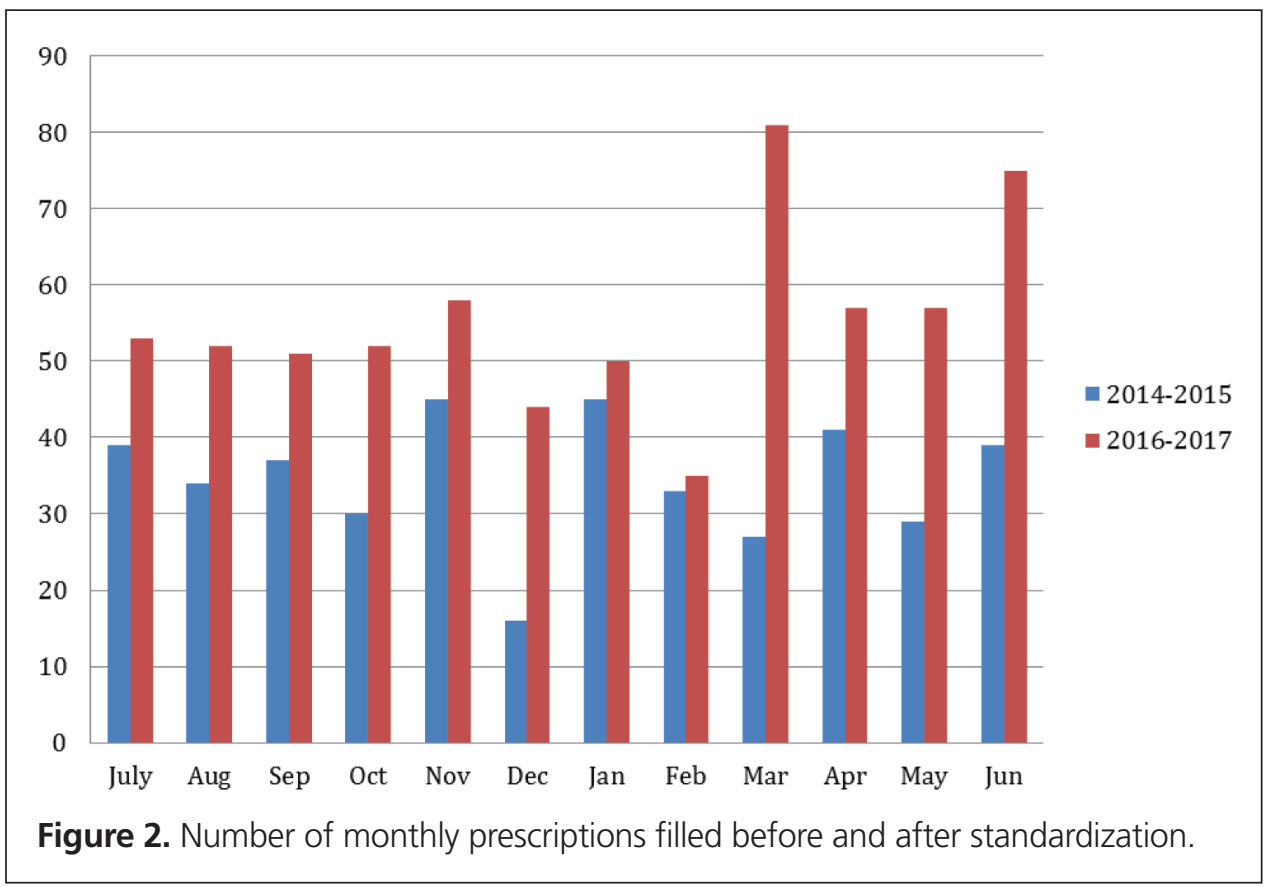

\section{DISCUSSION}

The importance of unresolved drug allergies in clinical care is well known, and the negative impact of such allergies during hospitalization has been extensively discussed. Most concerns regarding drug allergy pertain to antibiotics. ${ }^{5-7,14,17-23}$

Before the establishment of the drug allergy evaluation program at the study hospital, variability in physicians' approaches to drug allergy testing was identified. To optimize the service, activities were reviewed, with the aim of integrating pharmacy and allergy-related activities. It was agreed that a consensus-based, standardized, and transparent approach would be beneficial. This approach represented a major change in culture and required improved interdisciplinary collaboration and pooling of allergist resources. Recent literature has echoed the need for such multidisciplinary teams and systematic standardized approaches in evaluating suspected drug allergies. ${ }^{20}$

In the field of drug allergy testing, 2 important objectives are to achieve diagnostic accuracy and to maintain maximum safety. Guidelines helped to ensure safety and provided direction for standardization. Literature comparing the validity of various doses is limited and has been generated from retrospective data. ${ }^{14}$ About $10 \%$ of the general population claims to have an allergy to penicillin, yet more than $90 \%$ of these claims are eventually determined to be false. ${ }^{24}$ Because of the high prevalence of suspected drug allergy, it was important to develop a rapid yet safe triage process to both minimize risk and optimize workload. The rapid identification of patients with low probability of drug allergy was a key factor in optimizing pharmacy activities and in shortening evaluation time at the clinic. The triage tool and the standard operating procedures have been essential to these changes.

Workflow streamlining occurred primarily through a shift to use of single-dose challenges, accomplished by application of the triage (risk assessment) tool by all physicians. Application of this tool reduced the time required to conduct testing from $4 \mathrm{~h}$ to $90 \mathrm{~min}$ for patients at lower risk of a reaction. The ability to conduct more challenges per day and the inherent great increase in the number of prescriptions was offset by the availability of on-site bulk drug dispensing. This shift to single-dose testing improved patients' access by doubling or possibly tripling the number of patients tested daily. Monitoring pharmacy activities allowed us to identify the needs of the drug allergy clinic and to optimize pharmacy support by determining the drugs and doses most frequently used.

Several additional benefits emerged from this practice update. Using the risk assessment tool, allergists were able to cross-supervise drug challenges. The interchangeability of physicians to perform the evaluation and testing, as well as protocol simplification, facilitated the daily operation of multiple drug allergy clinics, including a clinic for general drug testing and a clinic specific for preoperative penicillin allergy "de-labelling". The number of half-day clinics per week increased from 2 to 6. Standardization also provided a more robust method to evaluate the impact of drug allergy testing on clinical outcomes and knowledge transfer. ${ }^{25}$ Optimizing the prophylactic use of antibiotics in surgery improved operating room efficiency. ${ }^{22}$ These encouraging results led to plans to extend testing to other regional health care centres and to target specific units with high rates of adverse reactions to drugs, such as the dialysis and oncology units. 
With any such expansion of the program, it will be necessary to reassess supporting pharmacy infrastructure. Through this program, we have identified a need to document clinical outcomes following de-labelling as part of a quality assurance platform. Quality improvement should go beyond assessing repeat drug exposure after de-labelling. It must include communication and reporting issues, safety assessment, and impact on long-term patient care.

Simplification and standardization of the allergy assessment were critical to the success of the program. Close interdisciplinary collaboration was important to minimize the impact on resources. The pharmacy infrastructure to support the allergy clinic was assigned to an existing pharmacy research department. Although the institution's department of pharmacy offers services to 3 sites, only one of these sites provides full allergy support. Specifically, one pharmacist and one pharmacy technical assistant at the Montreal General Hospital site of the McGill University Health Centre are assigned to support a sector that includes clinical drug research, management of drugs in the special access program, and provision of drug allergy testing. Following standardization, there was no increase in personnel to support the drug allergy program. Instead, there has been improved human resource utilization, which can be attributed to the simplification and standardization process that we employed.

An unexpected benefit of this quality improvement program was the review of internal and external pharmacy communication. The pharmacy record uniquely identified each patient and comprehensively documented details of the drug used in the challenge. Although the physician's report of the testing outcome was scanned and entered in the EMR, the drug allergy flag was not simultaneously removed from the EMR in all cases. All prescriptions were in a non-EMR pharmacy database, which allowed for rapid identification of those tested. The availability of duplicate documentation across databases proved useful as a means of identifying those instances where full de-labelling of the patients by the physicians was not completed in the EMR. This duplicate information may prove useful in developing a quality assurance program. Knowledge transfer relating to allergy labels has been identified as suboptimal in many institutions, including ours. ${ }^{6,22,25}$

There was a need to create a simple version of the final, allergist-certified report in the EMR, in addition to the source challenge documents. The above findings echo recommendations of others in the recent literature. ${ }^{6,26}$ Further improvements in communication are necessary, including uniform reporting terminology and systematic transmission to community physicians and pharmacists of information about patients with successful de-labelling of drug allergies. Given the current high prevalence of suspected drug allergy, a multi-hospital approach may be necessary, ${ }^{23}$ with the establishment of an integrated program involving the broad community of health care professionals, including community pharmacists. ${ }^{6}$

\section{CONCLUSION}

The safe administration of drugs is a prerequisite for excellence in patient care. It necessitates clarification of patients' drug allergy claims before drug administration. Standardizing and updating drug allergy testing had a beneficial effect on pharmacy compounding activities and led to improvement in patients' access to testing. The volume of prescriptions and the number of patients tested for drug allergy have increased substantially without any need for extra pharmacy staffing or equipment.

\section{References}

1. Sousa-Pinto B, Fonseca JA, Gomes ER. Frequency of self-reported drug allergy: a systematic review and meta-analysis with meta-regression. Ann Allergy Asthma Immunol. 2017;119(4):362-73.e2.

2. Thong BY, Tan TC. Epidemiology and risk factors for drug allergy. Br J Clin Pharmacol. 2011;71(5):684-700.

3. Lee CE, Zembower TR, Fotis MA, Postelnick MJ, Greenberger PA, Peterson LR, et al. The incidence of antimicrobial allergies in hospitalized patients: implications regarding prescribing patterns and emerging bacterial resistance. Arch Intern Med. 2000;160(18):2819-22.

4. van Dijk SM, Gardarsdottir H, Wassenberg MW, Oosterheert JJ, de Groot MC, Rockmann H. The high impact of penicillin allergy registration in hospitalized patients. J Allergy Clin Immunol Pract. 2016;4(5):926-31.

5. Picard M, Begin P, Bouchard H, Cloutier J, Lacombe-Barrios J, Paradis J, et al. Treatment of patients with a history of penicillin allergy in a large tertiary-care academic hospital. J Allergy Clin Immunol Pract. 2013;1(3):252-7.

6. Shaw BG, Masic I, Gorgi N, Kalfayan N, Gilbert EM, Barr VO, et al. Appropriateness of beta-lactam allergy record updates after an allergy service consult. J Pharm Pract. 2018:897190018797767.

7. Rubin R. Overdiagnosis of penicillin allergy leads to costly, inappropriate treatment. JAMA. 2018;320(18):1846-8.

8. Bernstein IL, Li JT, Bernstein DI, Hamilton R, Spector SL, Tan R, et al. Allergy diagnostic testing: an updated practice parameter. Ann Allergy Asthma Immunol. 2008;100(3 Suppl 3):S1-148.

9. Mirakian R, Ewan PW, Durham SR, Youlten LJ, Dugue P, Friedmann PS et al. BSACI guidelines for the management of drug allergy. Clin Exp Allergy. 2009;39(1):43-61.

10. Macy E, Romano A, Khan D. Practical management of antibiotic hypersensitivity in 2017. J Allergy Clin Immunol Pract. 2017;5(3):577-86.

11. Messaad D, Sahla H, Benahmed S, Godard P, Bousquet J, Demoly P. Drug provocation tests in patients with a history suggesting an immediate drug hypersensitivity reaction. Ann Intern Med. 2004;140(12):1001-6.

12. Fernandez TD, Ariza A, Palomares F, Montanez MI, Salas M, Martin-Serrano A, et al. Hypersensitivity to fluoroquinolones: the expression of basophil activation markers depends on the clinical entity and the culprit fluoroquinolone. Medicine (Baltimore). 2016;95(23):e3679.

13. Romano A, Gaeta F, Valluzzi RL, Caruso C, Rumi G, Bousquet PJ. IgEmediated hypersensitivity to cephalosporins: cross-reactivity and tolerability of penicillins, monobactams, and carbapenems. J Allergy Clin Immunol. 2010;126(5):994-9.

14. Iammatteo M, Blumenthal KG, Saff R, Long AA, Banerji A. Safety and outcomes of test doses for the evaluation of adverse drug reactions: a 5-year retrospective review. J Allergy Clin Immunol Pract. 2014;2(6):768-74.

15. Chiriac AM, Rerkpattanapipat T, Bousquet PJ, Molinari N, Demoly P. Optimal step doses for drug provocation tests to prove beta-lactam hypersensitivity. Allergy. 2017;72(4):552-61.

16. Shenoy ES, Macy E, Rowe T, Blumenthal KG. Evaluation and management of penicillin allergy: a review. JAMA. 2019;321(2):188-99.

17. Robitaille G, Karam F, Tardif M. Avis sur la standardisation des pratiques relatives aux allergies aux bêta-lactamines. Québec (QC): Gouvernement du Québec, INESSS [Institut national d'excellence en santé et en services sociaux]; 2017.

18. Satta G, Hill V, Lanzman M, Balakrishnan I. B-Lactam allergy: clinical implications and costs. Clin Mol Allergy. 2013;11(1):2. 
19. Sade K, Holtzer I, Levo Y, Kivity S. The economic burden of antibiotic treatment of penicillin-allergic patients in internal medicine wards of a general tertiary care hospital. Clin Exp Allergy. 2003;33(4):501-6.

20. Blumenthal KG, Ryan EE, Li Y, Lee H, Kuhlen JL, Shenoy ES. The impact of a reported penicillin allergy on surgical site infection risk. Clin Infect Dis. 2018;66(3):329-36.

21. Banks TA, Ressner RA, Gada SM. Antibiotic reclamation: penicillin allergy, antibiotic stewardship, and the allergist. Ann Allergy Asthma Immunol. 2015;115(5):451-2.

22. Moussa Y, Shuster J, Matte G, Sullivan A, Goldstein RH, Cunningham D, et al. De-labeling of beta-lactam allergy reduces intraoperative time and optimizes choice in antibiotic prophylaxis. Surgery. 2018;164(1):117-23.

23. Blumenthal KG, Shenoy ES, Wolfson AR, Berkowitz DN, Carballo VA, Balekian DS, et al. Addressing inpatient beta-lactam allergies: a multihospital implementation. J Allergy Clin Immunol Pract. 2017;5(3):616-25.e7.

24. Patterson RA, Stankewicz HA. Penicillin allergy. In: StatPearls. Treasure Island (FL): StatPearls Publishing; 2019.

25. Moussa Y, Sullivan A, Matte G, Goldstein RH, Baldini G, Shuster J, et al. Impact of persistent B-lactam allergy documentation despite delabeling in the perioperative setting. J Allergy Clin Immunol Pract. 2020;8(1):411-2.

26. New recommendations to improve drug allergy capture and clinical decision support. Horsham (PA): Institute for Safe Medication Practices (US); 2019

Gilbert Matte, BPharm, PhD, is with the Department of Pharmacy, McGill University Health Centre, Montréal, Quebec.

Joseph Shuster, MD, is with the Department of Medicine, Division of Allergy and Clinical Immunology, McGill University Health Centre, Montréal, Quebec.
Chantal Guevremont, BPharm, MSc, is with the Department of Pharmacy, McGill University Health Centre, Montréal, Quebec.

Phil Gold, MD, is with the Department of Medicine, Division of Allergy and Clinical Immunology, McGill University Health Centre, Montréal, Quebec.

Fabrice Leong, PharmD, is with the Department of Pharmacy, McGill University Health Centre, Montréal, Quebec.

Zinquon Ngan, PharmD, MSc, is with the Department of Pharmacy, McGill University Health Centre, Montréal, Quebec.

André Bonnici, BPharm, MSc, is with the Department of Pharmacy, McGill University Health Centre, Montréal, Quebec.

Chris Tsoukas, MD, is with the Department of Medicine, Division of Allergy and Clinical Immunology, McGill University Health Centre, Montréal, Quebec.

\section{Competing interests: None declared.}

\section{Address correspondence to:}

Dr Gilbert Matte

McGill University Health Centre

1650 Cedar Avenue, Room C1-200

Montréal QC H3G 1A4

e-mail: gilbert.matte@muhc.mcgill.ca

Funding: The Anna-Maria Solinas Laroche Allergy and Clinical Immunology Research Fund of the Montreal General Hospital Foundation provided funding for the preparation of this manuscript.

Acknowledgements: The authors would like to thank members and staff of the Division of Allergy and Clinical Immunology and the Department of Pharmacy of the McGill University Health Centre for their collaboration, as well as Debbie Cutler for manuscript editing in advance of submission.

\section{ON THE FRONT COVER}

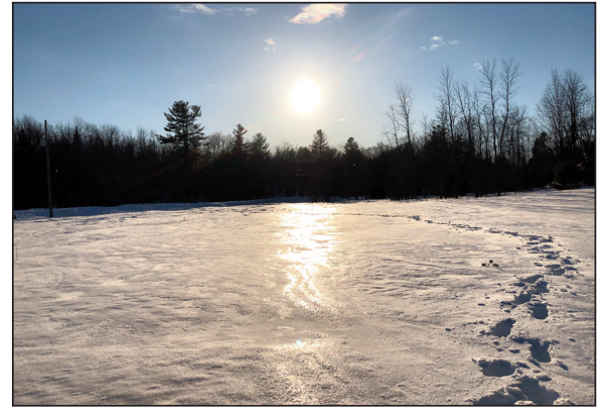

\section{Footprints in the Snow Carp Ridge, Ottawa, Ontario}

Amanda Iannaccio took this picture in rural Kanata in February 2018 with her iPhone 8. The photo captures Carp Ridge, a small range of rocky hills above the Carp Valley, one of the largest environmentally significant areas within the National Capital region. Amanda, who is CSHP's Content Officer, has been working in publishing for a decade and has been coordinating the publication of the Canadian Journal of Hospital Pharmacy for the past 5 years. She enjoys long walks in nature, listening to jazz on vinyl, and visiting wineries with her husband in their spare time.

The CJHP would be pleased to consider photographs featuring Canadian scenery taken by CSHP members for use on the front cover of the Journal. If you would like to submit a photograph, please send an electronic copy (minimum resolution $300 \mathrm{dpi}$ ) to publications@cshp.ca. 